\title{
Investigating compressed baryonic matter - the CBM experiment at FAIR
}

\author{
Claudia Höhne* \\ Justus-Liebig-Universität Giessen \\ for the CBM collaboration \\ E-mail: claudia.hoehne@physik.uni-giessen.de
}

\begin{abstract}
The CBM experiment at FAIR will investigate strongly interacting matter at high baryon densities in $\mathrm{A}+\mathrm{A}$ collisions from $2 A$ to $45 A \mathrm{GeV}$ beam energy (for $Z / A=0.5$ ). In the corresponding range of the QCD phase diagram only exploratory measurements have been performed so far, and lattice calculations are not applicable yet. On the other hand, model calculations predict new phases and structures such as a first order phase transition in the QCD phase diagram at large net-baryon densities. CBM will explore the created matter in particular with rare probes such as multi-strange hyperons, dileptons or charm, but also with event-by-event fluctuations of conserved quantities, and collective flow of identified particles. The experimental preparations in terms of detector development, feasibility studies and fast track reconstruction are progressing well such that CBM will be ready with the FAIR start.
\end{abstract}

9th International Workshop on Critical Point and Onset of Deconfinement - CPOD2014,

17-21 November 2014

ZiF (Center of Interdisciplinary Research), University of Bielefeld, Germany

\footnotetext{
* Speaker.
} 


\section{The CBM physics case}

The CBM experiment will investigate highly compressed strongly interacting matter created in A+A collisions from $2 A$ to $45 A \mathrm{GeV}$ beam energy at the FAIR accelerators SIS 100 and SIS 300. Nuclear matter and energy densities achieved in those heavy ion collisions have been calculated with various models [1] and are expected to reach up to approx. $5 \rho_{0}$ and $1.5 \mathrm{GeV} / \mathrm{fm}^{3}$ for $5 \mathrm{~A} \mathrm{GeV}$ and up to $10 \rho_{0}$ and $5 \mathrm{GeV} / \mathrm{fm}^{3}$ for $20 \mathrm{~A} \mathrm{GeV}$ beam energy. Fig. 1 (left) illustrates energy density and net-baryon density for one of the model calculations. The time where the density is larger than half the maximum lasts for at least $5 \mathrm{fm} / \mathrm{c}$. The structure and characteristics of matter at these densities are yet unknown and are in the focus of the CBM experiment. In the conjectured QCD
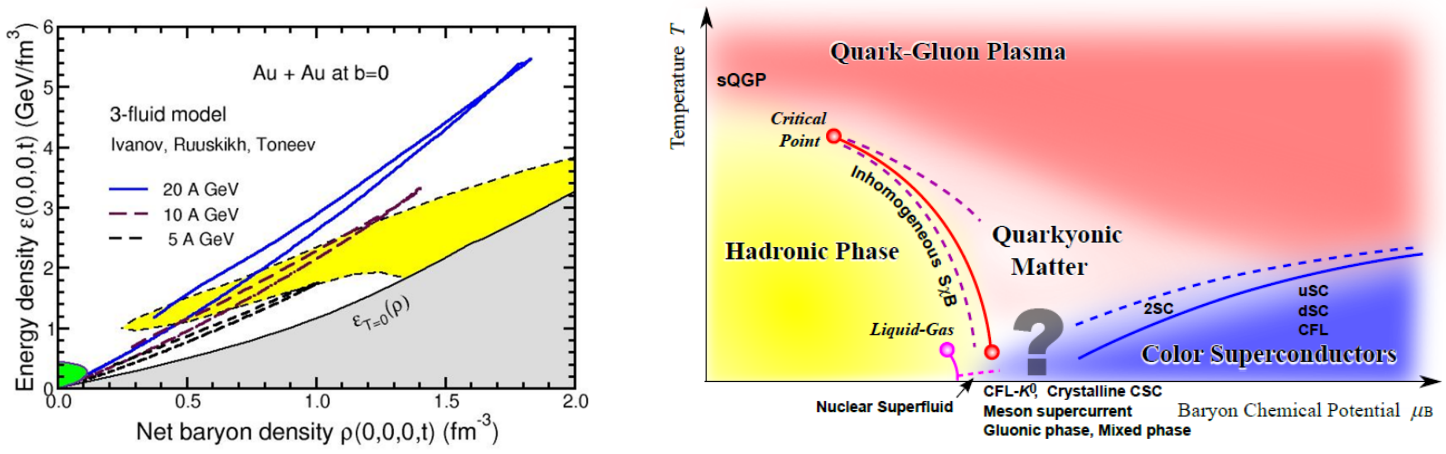

Figure 1: Left: Energy density versus net baryon density in the central cell of Au+Au collisions at various energies from a 3-fluid model [2]. The expected phase coexistence region is indicated schematically (yellow) while the chemical freeze-out (green) is based on fits to data. Right: Conjectured QCD phase diagram [3].

phase diagram as e.g. shown in one representation in Fig. 1 (right) [3], the high density part shows structures such as phase transition(s) which may end in a critical point, and possible new phases as the quarkyonic phase [4]. In order to investigate this largly unknown range of the QCD phase diagram, heavy-ion collisions of $2 A$ to $45 A \mathrm{GeV}$ beam energy are necessary at high intensities, and an experiment being able to cope with high interaction rates. This will allow the systematic and detailed investigation of rare probes such as dileptons, multi-strange particles and charm which are expected to be sensitive on characteristics of the created matter. The experience from RHIC and the investigation of the QCD phase diagram at low net-baryon densities has shown that we can expect new insight into high baryon density matter once new, high precision experimental data become available.

Reviewing the experimental situation until today, some evidence is seen for crossing a phase transition to deconfined matter for $30 \mathrm{~A} \mathrm{GeV}$ beam energy and above [5, 6, 7], and for entering a strongly coupled partonic phase at center of mass energies of $200 \mathrm{GeV}$ [8]. In particular the latter evidence is based on the measurement of a number of observables including multi-strange hyperons, dileptons, flow and correlations of identified particles. However, data for lower beam energies are scarce and so is the knowledge of the structure of matter at high baryon densities. For example, it is not clear whether strangeness is in equilibrium because no data for multi-strange particles exist, see the compilation in [7]. This question may be closely connected to whether or 
not hadronization takes place at a phase boundary [9]. Adding information on the flow of multistrange particles will shed light on the equation of state and dynamics of dense baryonic matter. Because of the high baryon density, a maximum in the production cross section of hypernuclei is expected [10], and also exotic strange particles may be produced [11]. No data exist at FAIR energies for fluctuations of any kind of variables or conserved quantities which may indicate phase transitions or a critical endpoint [12]. Electromagnetic probes are amongst the most promising ones characterizing any kind of strongly interacting matter as they give direct access to the fireball because of their penetrating nature. CBM will be the first experiment in the FAIR energy range being able to measure dileptons in the whole mass range from the photon point to $J / \psi$ in order to access a wide variety of physics: The measurement of direct photons via conversions for which the PHENIX collaboration showed a pioneering measurement [13] will give access to thermal radiation of the fireball. The strength of the dilepton yield at low masses has been shown to be strongly related to the coupling to baryons $[14,16,15]$. If quarkyonic matter exists at high baryon densities, dilepton radiation with particular characteristics may be a clear signature [17]. The intermediate mass range gives also access to fireball radiation [18] while a measurement of the $J / \psi$ probes the dense partonic matter on a high energy scale. Charm production will be investigated close to threshold, and it is expected that the production environment for charmed hadrons plays an important role for the fraction of charm quarks that end up in $\mathrm{J} / \psi$ or open charm. Being able to measure both, $\mathrm{J} / \psi$ and open charm, will give insight on the production scenario [1].

The physics program of CBM at SIS 100 and SIS 300 will address all above listed open questions. This unprecedented program of systematic studies of baryon dominated, high density matter in $\mathrm{A}+\mathrm{A}$ collisions will then give new insight into the structures of baryon dense matter and will allow to map the QCD phase diagram at high densities.

The running scenario of CBM foresees a start-up phase at SIS 100 in which beam energies from $2 A$ to $11 A \mathrm{GeV}$ will be provided, see Table 1 . At SIS 100, the HADES experiment will run

Table 1: Overview on accessible maximum beam energies at SIS100.

\begin{tabular}{lcc}
\hline Beam & $p_{\text {lab, } \max }$ & $\sqrt{s_{N N, \max }}$ \\
\hline heavy ions $(\mathrm{Au})$ & $11 A \mathrm{GeV} / \mathrm{c}$ & $4.7 \mathrm{GeV}$ \\
light ions $(Z / A=0.5)$ & $14 A \mathrm{GeV} / \mathrm{c}$ & $5.3 \mathrm{GeV}$ \\
protons & $29 \mathrm{GeV} / \mathrm{c}$ & $7.5 \mathrm{GeV}$ \\
\hline
\end{tabular}

as well, focussing on $2 A$ to $4 A \mathrm{GeV}$ beam energy. Therefore, currently in CBM an energy scan with $\mathrm{Au}+\mathrm{Au}$ and $\mathrm{C}+\mathrm{C}$ collisions at 4 energies $(4,6,8$, and $10 A \mathrm{GeV}$ beam energy) is planned. This program will then be followed up at the SIS 300 accelerator with the higher energy range up to $45 \mathrm{~A}$ $\mathrm{GeV}$ beam energy for $Z / A=0.5$. The complementarity of CBM and HADES in particular in terms of polar angle and material budget will offer a perfect handshake at $\mathrm{Au}+\mathrm{Au}$ collisions of about $2 \mathrm{~A}$ to $3 A \mathrm{GeV}$ beam energy.

\section{The CBM experiment}

The CBM experiment will measure the desired hadronic and leptonic probes with a wide phase 
space acceptance. The proposed full detector system is shown schematically in Figure 2. The core of CBM will be a silicon tracking system (STS) in a magnetic dipole field for tracking and momentum information. In order to investigate open charm production, an additional micro-vertex detector (MVD) is placed $5 \mathrm{~cm}$ behind the target. This setup is followed by detectors for particle identification: a RICH and a transition radiation detector (TRD) for electron identification, a time-of-flight (TOF) wall for hadron identification, and an electromagnetic calorimeter (EMC) for the measurement of direct photons in selected regions of phase space. Dimuon measurements will be possible by using an active absorber for muon identification $(\mathrm{MuCh})$, which is interchangeable with the RICH. This way CBM will have the opportunity for independent measurements of dielectron and dimuon pairs. Event classification, i.e. centrality and event plane determination will be performed by the projectile-spectator detector (PSD).

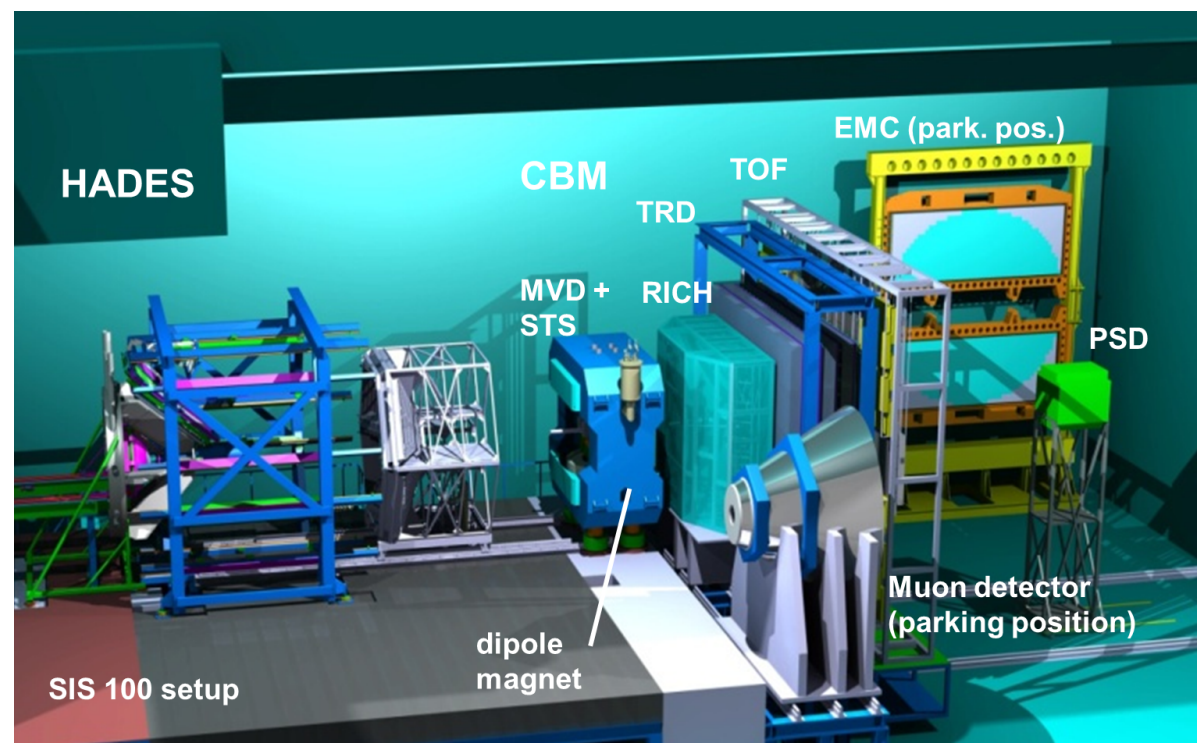

Figure 2: Setup of the HADES and CBM experiment at SIS 100; CBM with detectors for electron identification.

The CBM design as described above is optimized for the measurement of rare diagnostic probes at high rates: The reconstruction of multi-strange particles and open charm requires high precision tracking and a secondary vertex resolution in the range from $50 \mu \mathrm{m}$ to several $\mathrm{mm}$ depending on the lifetime of the hadron under investigation. A measurement of charmonium needs the ability of the whole detector to run at up to $10 \mathrm{MHz}$ interaction rates. Those two main experimental challenges have strong impact on the layout in particular close to the target but also on hardware and the online event selection concept. For open charm reconstruction a micro-vertex silicon pixel detector inside a magnetic field and just a few $\mathrm{cm}$ apart from the main vertex is a prerequisite. In order to allow for further high precision secondary vertex reconstruction as necessary for multi-strange hyperons, the MVD is followed by silicon strip detectors, still all inside a large magnetic field. Particle identification detectors then can only be added downstream: first for electrons (muons) and then for hadrons and neutral particles. As CBM is a fixed target experiment at "medium" energies, a large solid angle has to be covered for a wide phase space acceptance - here the choice is a polar angle coverage from $2.5^{\circ}-25^{\circ}$ limited by track density on the lower and detec- 
tor size on the upper side. In order to meet the requirement of high interaction rates, all detectors have to be fast and radiation hard. In CBM, the concept for fast readout and online event selection is based on self-triggered readout electronics shipping all data to a high performance computer cluster on which online event reconstruction and selection is performed in real-time.

\section{Detector development}

Detector development for CBM is progressing well, see Fig. 3 for a compilation of different developments and successfully tested mostly real-size prototypes. Technical Design Reports for Magnet, STS [19], RICH [20], MuCh, PSD and TOF have been approved. The remaining TDRs of MVD, DAQ/ FLES, TRD and EMC shall be submitted in 2015. In this article, only some broad overview on the various developments can be given. For detailed information on a specific detector development, the interested reader is referred to the detector articles given in the references.
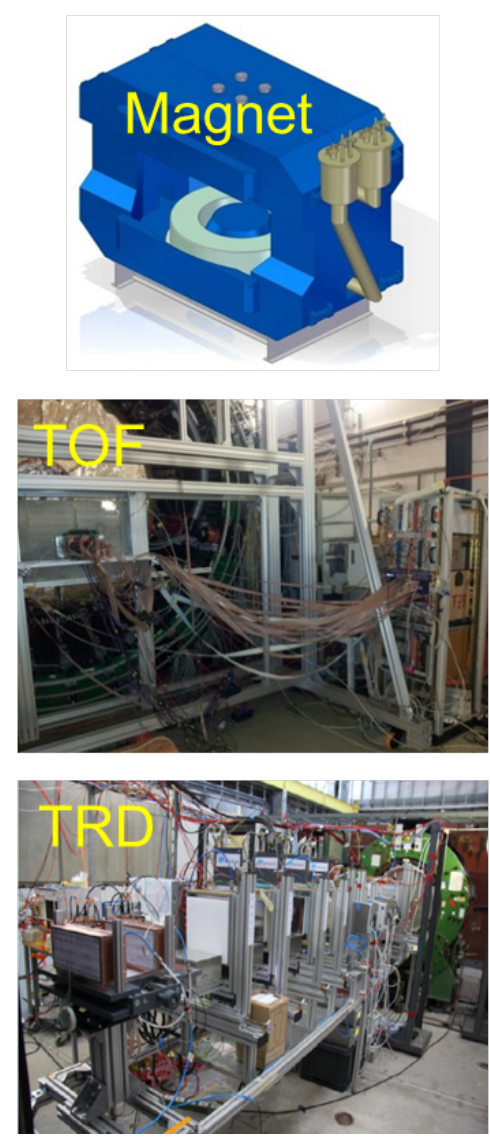
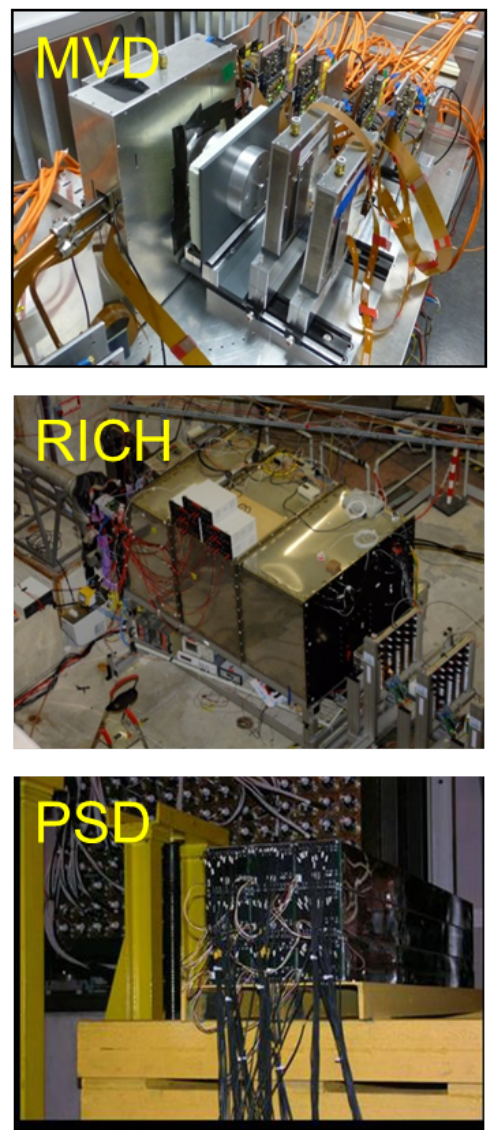
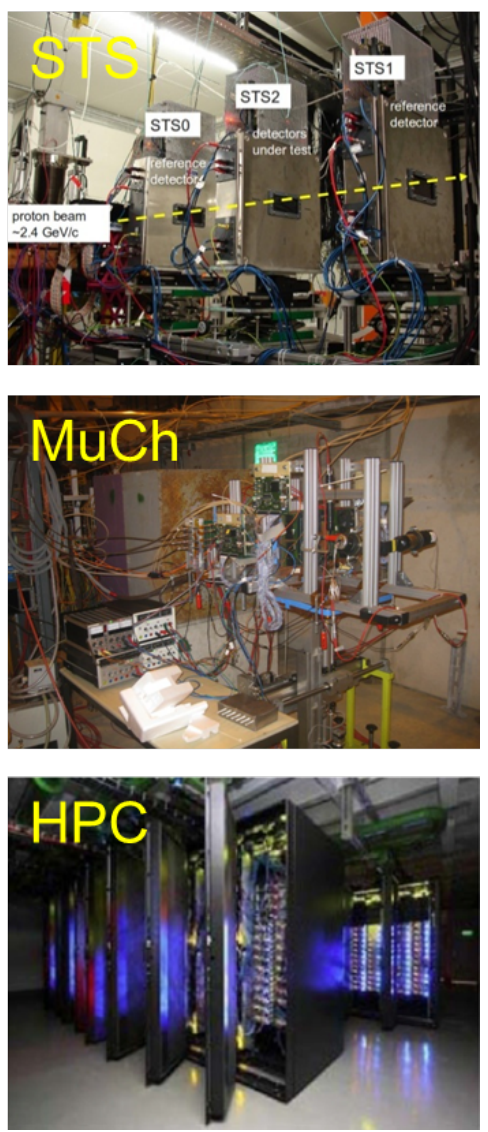

Figure 3: CBM technical developments : Technical drawing of the superconducting dipole magnet; MVD prototype in beamtest at CERN; STS sensor test at COSY; TOF prototypes under test at GSI; real size prototype of RICH in beamtest at CERN; GEM chambers of MuCh detector under test at CERN; large variety of different TRD chambers in beamtest at CERN; PSD moduls under test at CERN; HPC cluster as already realized at FIAS (from left to right and top to bottom).

The heart of CBM will be the silicon tracking system with radiation hard, double-sided silicon microstrip detectors with $7.5^{\circ}$ stereo angle and $58 \mu \mathrm{m}$ pitch. Single sensors will be $300 \mu \mathrm{m}$ thick 
and bonded to ultra-thin micro cables for readout at the periphery of the system, see [21] for more details. Highest and unambiguous track resolution close to the vertex for open charm reconstruction will be achieved by ultra-thin Si-pixel detectors. The development in CBM concentrates on using Monolythic Active Pixel Sensors (MAPS). Those sensors are routinely thinned down to $50 \mu \mathrm{m}$ and offer single point resolution of a few $\mu \mathrm{m}$ only, however, on account of having a rather long readout time and moderate radiation hardness. Both could be improved considerably in the past years, as for example reported in Ref. [22]. Electron identification detectors will be a gaseous RICH detector followed by 10 layers of transition radiation detectors. The RICH design comprises $\mathrm{CO}_{2}$ as radiator gas, spherical glass mirrors as focussing elements, and multi-anode photomultipliers for single photon detection [23]. In a real-size prototype tested at CERN-PS more than 20 hits per electron ring could be reconstructed at a noise level of approximately $10 \mathrm{~Hz}$ only. For the TRD, real dimension chambers were tested in the same testbeam campaigns and a large variety of radiator types (fibers, foils, foams) and chamber types ( $w \&$ w/o drift, different drift length, ...) were investigated [24]. In the last beamtime campaign at CERN-PS, November 2014, for the first time in CBM a new interface board (FLIB) developed for the first level event selection (FLES) was tested in a testbeam setup. This readout chain offers online and offline analysis via CbmRoot [25] and will further be developed to guarantee the required high throughput online analysis. The data structures in this readout (FLESnet) are already close to the final ones and the employed FLESnet delivers the desired time sliced objects. The MuCh detector will be an instrumented absorber where the first gaps will be equipped with GEM detectors because of the high particle rates [26]. For the absorber gaps further downstream straw tubes are foreseen. In order to instrument a large time-offlight detector at the end of the CBM setup at an affordable price, RPCs will be used [27]. Here, a lot of effort has successfully been made in order to provide high quality, low resistivity glasses of $10^{10}-10^{11} \Omega$ cm only.

\section{Feasibility studies and high performance computing}

All observables being relevant for the CBM physics program at SIS 100 and SIS 300 are studied in detail with the simulation framework CbmRoot [25] using GEANT 3 [28] for the detector simulations. Detectors were implemented mostly with realistic detector response functions adopted to testbeam data and including cluster finding algorithms. As event generator UrQMD v.3.3 [29] was used. For dileptons, signals were implemented using the PLUTO decayer [30] with multiplicities from HSD [31]. Complete event reconstruction with track reconstruction, secondary vertex finding and particle identification routines are implemented.

Some examples for recent simulation studies for SIS 100 and SIS 300 will be presented in the following. Fig. 4 shows feasibility studies for the reconstruction of hyperons at SIS 100. Assuming a storage rate of $100 \mathrm{kHz}$ about 500,000 $\Omega$-baryons will be measured per week allowing not only for the extraction of double-differential phase space distributions but also of flow and correlations. Fig. 5 and Fig. 6 show simulations for dielectrons and dimuons respectively, for more information see [32]. For the low-mass vector mesons cocktail-to-background ratios of $1 / 100$ are reached at invariant masses of $500 \mathrm{MeV} / \mathrm{c}^{2}$. Comparing with existing dilepton experiments, these conditions combined with the high statistics that CBM will deliver, will allow for high precision, multidifferential analysis of low-mass dilepton pairs. With anticipated storage rates of $100 \mathrm{kHz}$ close to $10^{6}$ 


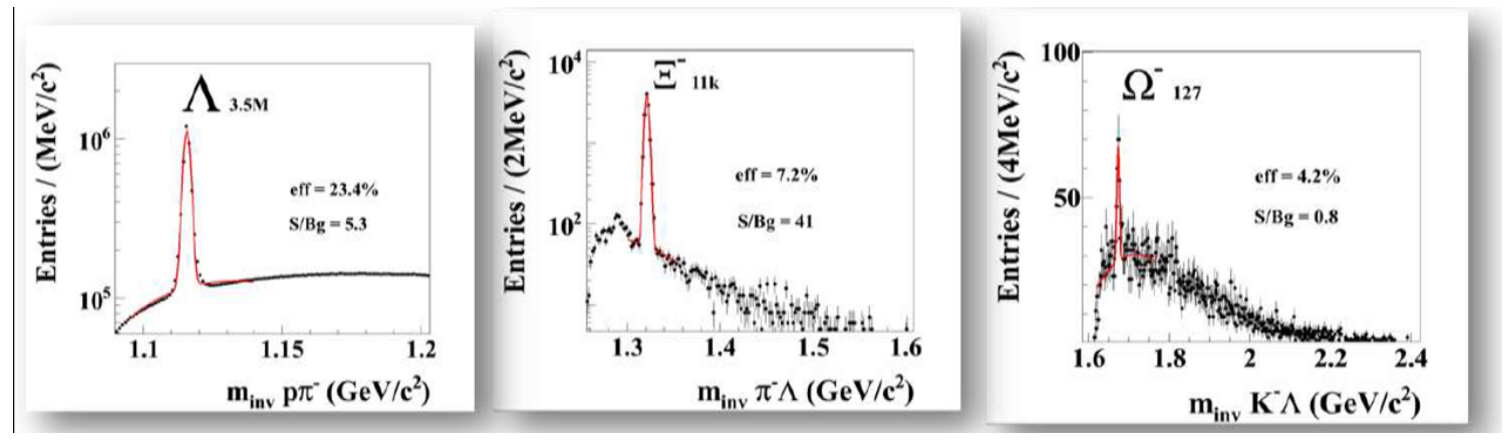

Figure 4: Feasibility studies of hyperon reconstruction in $1 \mathrm{M}$ central $(\mathrm{b}=0 \mathrm{fm}) \mathrm{Au}+\mathrm{Au}$ collisions at $8 A$ GeV beam energy.

$\omega$ mesons will be measured per week. The measurement of the $J / \psi$ at SIS 100 will be a first high rate challenge; with $10 \mathrm{MHz}$ interaction rate close to $10^{4} \mathrm{~J} / \psi$ will be measurable in the dimuon decay channel.
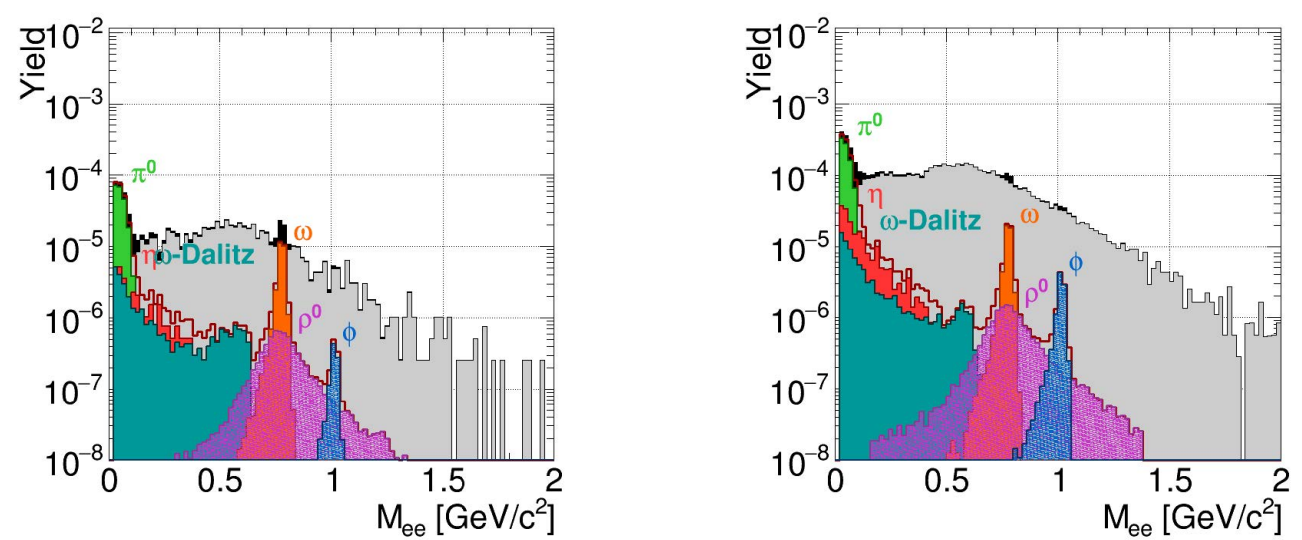

Figure 5: Simulated invariant mass spectra for low-mass vector mesons in the dielectron channel for $1 \mathrm{M}$ central $(b=0 \mathrm{fm}) \mathrm{Au}+\mathrm{Au}$ collisions at $8 A \mathrm{GeV}$ (left) and $25 A \mathrm{GeV}$ beam energy (right). The cocktail contains no in-medium contributions. Contributing sources for the cocktail of low-mass pairs are (from left to right): $\pi^{0}$-Dalitz, $\omega$-Dalitz, $\eta$-Dalitz, $\rho, \omega, \phi$.

A lot of effort is going towards the development of a high speed first level event selection (FLES) package [33] in order to meet the specific challenges in computing issues [34]. Those mainly arise from the quest of having online event reconstruction and selection including secondary vertex finding at reaction rates of $10^{5}-10^{7} \mathrm{~Hz}$. In CBM, the self-triggered readout electronics will deliver a stream of time-stamped data which will be analyzed on fast and large computer farms in order to deliver online event selection in real-time [35]. All tracking codes developed so far have been ported to modern multi- and manycore machines, and full use is made of parallel computing [36]. A standalone FLES package has been established that is portable, efficient, SIMDized (Single Instruction, Multiple Data), and fully parallelized. Its scalability has successfully been tested up to 80 cores on a single machine. Currently, with this package tracking in the STS and secondary 

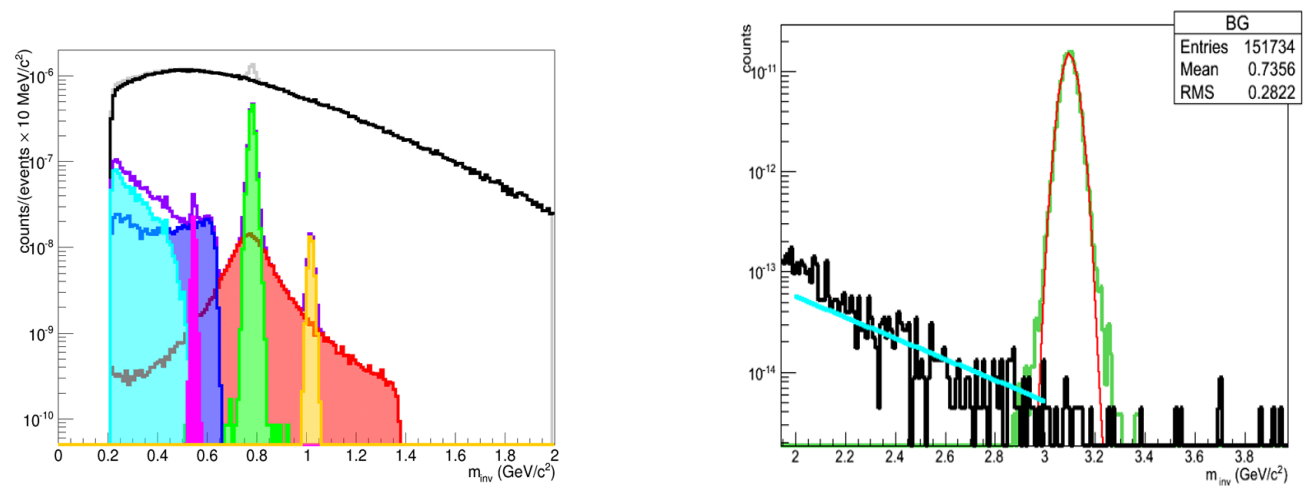

Figure 6: Simulated invariant mass spectra in the dimuon channel for low-mass vector mesons in central $(\mathrm{b}=0 \mathrm{fm}) \mathrm{Au}+\mathrm{Au}$ collisions at $8 \mathrm{~A} \mathrm{GeV}$ beam energy (left) and the $J / \psi$ in $\mathrm{p}+\mathrm{Au}$ collisions at $30 \mathrm{~A} \mathrm{GeV}$ beam energy (right). The cocktail contains no in-medium contributions. Contributing sources for the cocktail of low-mass pairs are (from left to right): $\omega$-Dalitz, $\eta$-Dalitz, $\eta, \rho, \omega, \phi$.

vertex finding takes only $8 \mathrm{~ms} /$ core for minimum bias and $62 \mathrm{~ms} /$ core for central Au+Au collisions at $25 \mathrm{~A} \mathrm{GeV}$ beam energy. New developments of the tracking algorithms include time as a forth dimension in order to prepare for event reconstruction from free streaming readout that delivers hits with a time stamp. Reconstructed tracks are then clustered in groups representing the original events. Important checks ongoing are the verification that no event splitting and event mergers occur.

\section{Summary}

Understanding the QCD phase diagram at high baryon densities needs systematic measurements in particular also of rare observables such as multi-strange hyperons, dileptons and charm. Measuring these probes, the CBM experiment will offer unprecedented possibilities to map and explore the QCD phase diagram at high net-baryon densities. CBM will thus offer the chance to resolve many open physics questions related to high density matter such as for the equation-of-state, phase transitions, new phases and in-medium modifications of hadrons and thus has a substantial discovery potential.

Experimental preparations in terms of detector development and high performance computing are proceeding well and will allow CBM to start taking data with the first beams delivered from SIS 100.

Acknowledgement This work was supported by the Hessian LOEWE initiative through the Helmholtz International Center for FAIR (HIC for FAIR), by the GSI F\&E-Cooperation with Giessen, and by BMBF grant 05P12RGFCG.

\section{References}

[1] B. Friman et al. (Eds), The CBM Physics Book: Compressed Baryonic Matter in Laboratory Experiments, Springer Series: Lecture Notes in Physics, Vol. 814 (2011). 
[2] Y.B. Ivanov, V.N. Ruuskikh, V.D. Toneev, Phys. Rev. C 73, 44904 (2006).

[3] K. Fukushima, T. Hatsuda, Rept. Prog. Phys.74:014001,2011.

[4] L. McLerran and R. Pisarski, Nucl. Phys. A 796 (2007) 83.

[5] C. Höhne, Nucl. Phys. A 830 (2009) 369c.

[6] C. Alt et al. [NA49 collaboration], Phys. Rev. C 77, 024903 (2008).

[7] A. Andronic et al., Phys. Lett. B 673 (2009) 142.

[8] RHIC white papers: First Three Years of Operation of RHIC, Nucl. Phys. A 757 (2005) 1-284.

[9] P. Braun-Munzinger, J. Stachel, C. Wetterich, Phys.Lett. B596 (2004) 61-69.

[10] A. Andronic, Phys. Lett. B 697 (2011) 203.

[11] H. Stöcker et al., Nucl. Phys. A 827 (2009) 624c.

[12] V. Koch et al., AIP Conf.Proc. 1520 (2013) 232-237; V. Koch, J.Phys. G35 (2008) 104030, V. Koch, arXiv:0810.2520.

[13] S. Afanasiev et al. [PHENIX collaboration], Phys. Rev. Lett. 109 (2012) 152302.

[14] G. Agakishiev et al. [HADES], Phys. Rev. C 84 (2011) 014902.

[15] D. Adamova et al. [CERES], Phys. Lett. B 666 (2008) 425.

[16] H. v.Hees and R. Rapp, Nucl. Phys. A 806 (2008) 339.

[17] G. Torrieri, S. Vogel, S. Lottini, J.Phys.Conf.Ser. 509 (2014) 012035.

[18] R. Arnaldi et al. [NA60], Eur. Phys. J C 61 (2009) 711.

[19] J. Heuser (Ed) et al., STS-TDR, GSI-2013-05499, http://repository.gsi.de/record/54798.

[20] C. Höhne (Ed) et al., RICH-TDR, GSI-2014-00528, http://repository.gsi.de/record/65526.

[21] I. Sorokin et al., Nucl. Instr. Meth. A 718 (2013) 366; J. Heuser, GSI DOC-2012-Feb-19.

[22] M. Deveaux at al., JINST 6 (2011) C02004, M. Deveaux at al., Nucl. Instr. Meth. A 718 (2013) 305; M. Koziel et al., Nucl. Instr. Meth. A 732 (2013) 515.

[23] J. Adamczewski et al., JINST 9 (2014) C06002; C. Höhne et al., Nucl. Instr. Meth. A 639 (2011) 294-297; C. Höhne et al., Nucl. Instr. Meth. A. 595 (2008) 187; J. Adamczewski et al., Nucl. Instr. Meth. A 766 (2014) 101.

[24] A. Arend et al., J.Phys.Conf.Ser. 426 (2013) 012022.

[25] M. Al-Turany et al., J. Phys.: Conf. Series 396 (2012) 022001.

[26] S. Biswas et al., JINST 8 (2013) C12002.

[27] J. Wang et al., Nucl. Instr. Meth. A 621 (2010) 151; J. Wang et al., Nucl. Instr. Meth. A 713 (2013) 40; I. Deppner et al., JINST 9 (2014) C10014.

[28] GEANT - Detector Description and Simulation Tool. CERN Program Library Long Writeup W5013.

[29] S. Bass et al., Prog. Part. Nucl. Phys. 41 (1998) 255.

[30] I. Froehlich et al., PoS ACAT2007 (2007) 076.

[31] W. Cassing, E. Bratkovskaya, Phys. Reports 308 (1999) 65. 
[32] C. Höhne, Nucl. Phys. A 931 (2014) 735.

[33] J. de Cuveland and V. Lindenstruth, J. Phys.: Conf. Ser. 331 (2011) 022006.

[34] V. Friese, Springer Lecture Notes in Computer Science, Volume 7125 (2012) 17.

[35] V. Friese, J. Phys.: Conf. Ser. 331 (2011) 032008.

[36] I. Kisel, J.Phys.Conf.Ser. 523 (2014) 012022. 